\title{
Efficacy and safety of evolocumab in individuals with type 2 diabetes mellitus: primary results of the randomised controlled BANTING study
}

\author{
Robert S. Rosenson ${ }^{1} \cdot$ Martha L. Daviglus ${ }^{2} \cdot$ Yehuda Handelsman $^{3} \cdot$ Paolo Pozzilli $^{4} \cdot$ Harold Bays $^{5}$. \\ Maria Laura Monsalvo ${ }^{6} \cdot$ Mary Elliott-Davey $^{7} \cdot$ Ransi Somaratne $^{6} \cdot$ Peter Reaven $^{8}$
}

Received: 20 November 2018 / Accepted: 18 February 2019 / Published online: 5 April 2019

(C) The Author(s) 2019

\begin{abstract}
Aims/hypothesis The study aimed to examine the efficacy of 12 weeks of monthly evolocumab or placebo in lowering LDLcholesterol (LDL-C) in individuals with type 2 diabetes and hypercholesterolaemia or mixed dyslipidaemia and on a maximumtolerated statin of at least moderate intensity.

Methods For this randomised, placebo-controlled outpatient study, eligible individuals were $\geq 18$ years old with type 2 diabetes, $\mathrm{HbA}_{1 \mathrm{c}}<10 \%$ ( $\left.86 \mathrm{mmol} / \mathrm{mol}\right)$, had been on stable pharmacological therapy for diabetes for $\geq 6$ months and were taking a maximum-tolerated statin dose of at least moderate intensity. Lipid eligibility criteria varied by history of clinical cardiovascular disease. Participants were randomised 2:1 to evolocumab $420 \mathrm{mg}$ s.c. or placebo. Randomisation was performed centrally via an interactive web-based or voice recognition system. Allocation was concealed using the centralised randomisation process. Treatment assignment was blinded to the sponsor study team, investigators, site staff and patients throughout the study. Coprimary endpoints were mean percentage change in LDL-C from baseline to week 12 and to the mean of weeks 10 and 12. Additional endpoints included LDL-C $<1.81 \mathrm{mmol} / 1$, LDL-C reduction $\geq 50 \%$ and other lipids. Exploratory analyses included percentage changes in fasting and post mixed-meal tolerance test (MMTT) lipoproteins and lipids, glucose metabolism variables and inflammatory biomarkers.

Results In total, 421 individuals were randomised and analysed, having received evolocumab (280 participants) or placebo (141 participants) (mean [SD] age 62 [8] years; 44\% women; 77\% white). Evolocumab decreased LDL-C by 54.3\% (1.4\%) at week 12 (vs $1.1 \%$ [1.9\%] decrease with placebo; $p<0.0001$ ) and by $65.0 \%(1.3 \%)$ at the mean of weeks 10 and 12 (vs $0.8 \%$ [1.8\%] decrease with placebo; $p<0.0001$ ); it also decreased non-HDL-cholesterol (HDL-C) by $46.9 \%$ (1.3\%) at week 12 (vs $0.6 \%$ [1.8\%] decrease with placebo) and by $56.6 \%(1.2 \%)$ at the mean of weeks 10 and 12 (vs $0.1 \%$ [1.6\%] decrease with placebo). Evolocumab significantly improved levels of other lipids and allowed more participants to reach LDL-C $<1.81 \mathrm{mmol} / 1$ or a reduction in LDL-C levels $\geq 50 \%$. After an MMTT (120 min), there were favourable changes $(p<0.05$; nominal, post hoc, no multiplicity adjustment) in chylomicron triacylglycerol (triglycerides), chylomicron cholesterol, VLDL-C and LDL-C. Evolocumab had no effect on glycaemic variables and was well tolerated.
\end{abstract}

Electronic supplementary material The online version of this article (https://doi.org/10.1007/s00125-019-4856-7) contains peer-reviewed but unedited supplementary material, which is available to authorised users.

Robert S. Rosenson

robert.rosenson@mssm.edu

1 Icahn School of Medicine at Mount Sinai, 1425 Madison Ave, MC Level, New York, NY 10029, USA

2 University of Illinois at Chicago College of Medicine, Chicago, IL, USA

3 Metabolic Institute of America, Tarzana, CA, USA
4 University Campus Bio-medico, Rome, Italy

5 Louisville Metabolic and Atherosclerosis Research Center, Louisville, KY, USA

6 Amgen Inc., Thousand Oaks, CA, USA

7 Amgen Ltd, Cambridge, UK

8 University of Arizona College of Medicine, Phoenix VA Health Care System, Phoenix, AZ, USA 


\section{Research in context}

\section{What is already known about this subject?}

- Diabetes mellitus is associated with an increased risk for cardiovascular disease morbidity and mortality

- In a post hoc analysis from the FOURIER trial, evolocumab treatment was associated with similar reductions in both $\mathrm{LDL}$-cholesterol (LDL-C) level and the risk of incident cardiovascular events in individuals with or without diabetes

\section{What is the key question?}

- In individuals with type 2 diabetes mellitus on maximally tolerated statin therapy, does 12 weeks of monthly evolocumab therapy sufficiently lower LDL-C without notable safety findings?

\section{What are the new findings?}

- Evolocumab decreased LDL-C by $54-65 \%$, decreased non-HDL-cholesterol by $47-57 \%$, significantly improved levels of other lipids and allowed more individuals to reach $\mathrm{LDL}-\mathrm{C}<1.81 \mathrm{mmol} / \mathrm{l}$ or a reduction in $\mathrm{LDL}-\mathrm{C}$ level $\geq 50 \%$

- Treatment with evolocumab led to favourable changes $(p<0.05)$ in postprandial levels of chylomicrons, VLDLcholesterol and LDL-C

\section{How might this impact on clinical practice in the foreseeable future?}

- These additional, placebo-controlled data support the efficacy and safety of evolocumab therapy in individuals with type 2 diabetes mellitus and hyperlipidaemia or mixed dyslipidaemia

Conclusions/interpretation In statin-treated individuals with type 2 diabetes and hypercholesterolaemia or mixed dyslipidaemia, evolocumab significantly reduced LDL-C and non-HDL-C. Favourable changes $(p<0.05)$ were observed in postprandial levels of chylomicrons, VLDL-C and LDL-C.

Trial registration ClinicalTrials.gov NCT02739984

Funding This study was funded by Amgen Inc.

Data availability Qualified researchers may request data from Amgen clinical studies. Complete details are available at www. amgen.com/datasharing.

Keywords Diabetes $\cdot$ Diabetic dyslipidaemia $\cdot$ Hypercholesterolaemia $\cdot$ Lipid-lowering therapy $\cdot$ PCSK9 inhibition

$\begin{array}{ll}\text { Abbreviations } & \\ \text { ACC } & \text { American College of Cardiology } \\ \text { AE } & \text { Adverse event } \\ \text { AHA } & \text { American Heart Association } \\ \text { ApoB } & \text { Apolipoprotein B } \\ \text { ApoB-48 } & \text { Apolipoprotein B48 } \\ \text { ASCVD } & \text { Atherosclerotic cardiovascular disease } \\ \text { BANTING } & \text { EvolocumaB efficAcy aNd safeTy IN type 2 } \\ & \text { diabetes mellitus on backGround statin therapy } \\ \text { COPD } & \text { Chronic obstructive pulmonary disease } \\ \text { CVD } & \text { Cardiovascular disease } \\ \text { FOURIER } & \text { Further Cardiovascular Outcomes Research } \\ & \text { With PCSK9 Inhibition in Subjects With } \\ & \text { Elevated Risk } \\ \text { HDL-C } & \text { HDL-cholesterol } \\ \text { LDL-C } & \text { LDL-cholesterol } \\ \text { Lp(a) } & \text { Lipoprotein(a) } \\ \text { MI } & \text { Myocardial infarction } \\ \text { MMTT } & \text { Mixed-meal tolerance test }\end{array}$

$\begin{array}{ll}\text { PCSK9i } & \begin{array}{l}\text { Proprotein convertase subtilisin/kexin type } 9 \\ \text { inhibitor }\end{array} \\ \text { Q } & \text { Quartile } \\ \text { VLDL-C } & \text { VLDL cholesterol }\end{array}$

\section{Introduction}

The prevalence of diabetes mellitus has increased progressively worldwide over the past several decades [1,2], and diabetes mellitus is associated with an increased risk for cardiovascular disease (CVD) morbidity and mortality [3, 4]. Individuals with diabetes mellitus who experience an acute myocardial infarction (MI) are at higher risk for recurrent cardiovascular events and mortality compared with their counterparts without diabetes mellitus [3, 4], and thus require an aggressive treatment approach [5-7]. In addition, many statin-treated individuals with type 2 diabetes have poorly controlled LDLcholesterol (LDL-C) and non-HDL-cholesterol (non-HDL- 
C) levels [7]. In the Further Cardiovascular Outcomes Research With PCSK9 Inhibition in Subjects With Elevated Risk (FOURIER) trial (ClinicalTrials.gov NCT01764633), the use of evolocumab $140 \mathrm{mg}$ every 2 weeks or $420 \mathrm{mg}$ once every month was associated with similar reductions in both LDL-C levels and the risk of incident cardiovascular events in individuals with or without diabetes [8].

The evolocumaB efficAcy aNd safeTy IN type 2 diabetes mellitus on backGround statin therapy (BANTING) study (NCT02739984) aimed to examine the efficacy of a 12 week regimen of s.c. evolocumab $420 \mathrm{mg}$ once monthly compared with placebo in lowering LDL-C and improving other lipid levels in individuals with type 2 diabetes and hypercholesterolaemia or mixed dyslipidaemia taking maximally tolerated background statin therapy of at least moderate intensity.

\section{Methods}

The primary objective of this study was to evaluate the effect of 12 weeks of treatment with s.c. monthly evolocumab compared with monthly placebo on percentage change in LDL-C among individuals with type 2 diabetes and hypercholesterolaemia or mixed dyslipidaemia on a maximally tolerated dose of statin of at least moderate intensity. The secondary objectives were to assess the effects of 12 weeks of treatment with monthly evolocumab compared with monthly placebo on: (1) change in LDL-C from baseline and the percentage change in non-HDL-C, apolipoprotein B (ApoB), total cholesterol, lipoprotein(a) (Lp(a)), triacylglycerol (triglycerides), HDL-C and VLDL cholesterol (VLDL-C); (2) percentage of participants achieving LDL-C $<1.81 \mathrm{mmol} / \mathrm{l}$; and (3) percentage of participants achieving a $\geq 50 \%$ reduction in LDL-C from baseline. The safety and tolerability of evolocumab were also assessed. Exploratory analyses included percentage changes in fasting, $120 \mathrm{~min}$ and $180 \mathrm{~min}$ post mixed-meal lipoprotein and glucose metabolism measures, and the inflammatory biomarkers adiponectin and IL-6.

Eligible individuals were $\geq 18$ years of age with type 2 diabetes, had $\mathrm{HbA}_{1 \mathrm{c}}<10 \%$ ( $86 \mathrm{mmol} / \mathrm{mol}$ ), were receiving stable pharmacological therapy for diabetes for $\geq 6$ months, and were taking a maximally tolerated dose of statin of at least moderate intensity (per the American College of Cardiology [ACC]/American Heart Association [AHA] definition). Eligibility criteria for LDL-C or non-HDL-C level varied depending on prior clinical CVD, defined as a history of MI, stable or unstable angina, coronary or other arterial revascularisation, stroke, transient ischaemic attack or peripheral arterial disease presumed to be of atherosclerotic origin. Individuals without known clinical CVD were required to have a fasting LDL-C during lipid stabilisation of $\geq 2.59 \mathrm{mmol} / \mathrm{l}$ or non-HDL-C $\geq 3.39 \mathrm{mmol} / \mathrm{l}$. Individuals with known clinical CVD were required to have a fasting LDL-C during lipid stabilisation of $\geq 1.81 \mathrm{mmol} / 1$ or non-HDL-C $\geq 2.59 \mathrm{mmol} / 1$. Individuals were randomised $2: 1$ to two treatment groups (evolocumab and placebo). Randomisation was performed centrally via an interactive web-based or voice recognition system. Allocation was concealed using the centralised randomisation process. Treatment assignment was blinded to the sponsor study team, investigators, site staff, and patients throughout the study. Randomisation was stratified by LDL-C (above or below $3.36 \mathrm{mmol} / \mathrm{l}$ ).

The co-primary endpoints were mean percentage change in LDL-C from baseline to week 12 and the mean percentage change in LDL-C from baseline to the mean of weeks 10 and 12. In this study, evolocumab was dosed at day 1 , week 4 and week 8 . Thus, week 12 was at the end of the dosing window. As maximum reduction of LDL-C occurs approximately 2 weeks post dose for individuals receiving evolocumab $420 \mathrm{mg}$ monthly [9], LDL-C would be measured at week 10, before LDL-C levels began to return to baseline. Thus, an average measure between weeks 10 and 12 was also taken for lipid measures. Secondary lipid endpoints for these same time periods included: change from baseline in LDL-C, percentage change from baseline in non-HDL-C, ApoB, total cholesterol, Lp(a), triacylglycerol, HDL-C and VLDL-C; achievement of LDL-C $<1.81 \mathrm{mmol} / \mathrm{l}$; and $\geq 50 \%$ reduction in LDL-C from baseline.

Exploratory analyses included percentage changes in AUC in fasting, $120 \mathrm{~min}$ and $180 \mathrm{~min}$ mixed-meal tolerance test (MMTT) lipoproteins and lipids, glucose metabolism variables and inflammatory biomarkers. For the MMTT, following an overnight fast, participants were fed a standardised liquid mixed meal. Meals could differ between study sites but were required to contain the same amount (component weight not differing by more than $\pm 15 \%$ ) of energy $(1004.16 \mathrm{~kJ}$ [240 kcal]), protein $(10 \mathrm{~g})$, total fat $(4 \mathrm{~g})$ and carbohydrate $(41 \mathrm{~g})$. The same type of standard mixed meal was required to be used for day 1 and week 12. Baseline and $2 \mathrm{~h}$ ( $\pm 10 \mathrm{~min}$ ) blood collection was done after the meal. In addition, a subset of individuals participated in MMTT extended-timepoint assessments, with three additional postprandial blood draws at $30 \mathrm{~min}( \pm 10 \mathrm{~min}), 1 \mathrm{~h}( \pm 10 \mathrm{~min})$ and $3 \mathrm{~h}( \pm 10 \mathrm{~min})$. AUCs for $0-120 \mathrm{~min}$ and for $0-180 \mathrm{~min}$ were calculated for the MMTT at day 1 and at week 12 for laboratory variables. Lipid and lipoprotein measures included apolipoprotein B48 (ApoB-48), chylomicron triacylglycerol and cholesterol, total cholesterol, LDL-C, VLDL-C, nonHDL-C, triacylglycerol and HDL-C. Measures of glucose metabolism included plasma glucose, insulin, proinsulin, C-peptide, glucagon and NEFA. Anti- and proinflammatory biomarkers were adiponectin and IL-6, respectively.

LDL-C was calculated using the Friedewald formula; however, if calculated LDL-C was $<1.03 \mathrm{mmol} / \mathrm{l}$ or triacylglycerol $>4.52 \mathrm{mmol} / \mathrm{l}$, then LDL-C was measured by ultracentrifugation from the same blood sample if additional adequate sample 
was available. VLDL-C was also calculated; similarly, if calculated LDL-C was $<1.03 \mathrm{mmol} / 1$ or triacylglycerol $>4.52 \mathrm{mmol} / \mathrm{l}$, VLDL-C was quantified via ultracentrifugation. Chylomicron triacylglycerol and cholesterol were measured by photometry after ultracentrifugation. All routine lipids, lipoproteins and inflammatory biomarkers were assayed in serum samples by MedPace (Cincinnati, OH, USA and Leuven, Belgium). Serum glucose and $\mathrm{HbA}_{1 \mathrm{c}}$ were assessed as part of chemistry, which was performed by $\mathrm{Q}^{2}$ Solutions (Valencia, CA, USA and Livingston, Scotland, UK). An institutional review board or independent ethics committee reviewed and approved this study and the study amendment at each study centre. This amendment entailed minor language changes that provided clarification for study centres and is not expected to have altered results. The investigator collected informed consent from all participants before any screening procedures were performed. This study was conducted in accordance with International Council for Harmonisation Good Clinical Practice regulations/guidelines.

Statistical analysis Safety and efficacy analyses were performed on all participants included in the full analysis set (all individuals who were randomised and received at least one dose of evolocumab or placebo; Fig. 1). A repeatedmeasures linear effects model was used to compare the efficacy of evolocumab vs placebo. This model includes terms for treatment group, pre-specified stratification by LDL-C level $(<3.36 \mathrm{mmol} / 1 \mathrm{vs} \geq 3.36 \mathrm{mmol} / \mathrm{l})$, scheduled visit and the interaction of treatment with scheduled visit. Missing values were not imputed. A Cochran-Mantel-Haenszel test adjusted by stratification factor was used to analyse LDL-C level achievement and LDL-C response. For testing, non-response was imputed for individuals with a missing value.

The adjusted $p$ values were calculated based on the multiplicity-testing strategy depicted in electronic supplementary material (ESM) Fig. 1; a $p$ value of 0.05 was used for comparative purposes to determine statistical significance. An overall family-wise error rate of 0.05 was maintained for all co-primary and co-secondary efficacy outcome testing using a combination of sequential testing, the fall-back procedure and the Hochberg procedure. All analyses were performed using SAS version 9.4 (SAS Institute, Cary, NC, USA).

\section{Results}

Participants A total of 421 individuals were randomised and received evolocumab $(n=280)$ or placebo $(n=141)$. The mean (SD) age was $62.5(8.5)$ years for evolocumab and 62.2 (8.4) years for placebo; $42.9 \%$ (evolocumab) and $46.1 \%$ (placebo) of participants were female; $79.3 \%$ (evolocumab) and $71.6 \%$ (placebo) of participants were white (Table 1). Baseline clinical characteristics, including systolic BP, BMI, waist circumference and background lipid- and diabetes-related medication use were similar between evolocumab and placebo groups. The mean (SD) baseline
Fig. 1 CONSORT flowchart of study design. Numbers of participants who completed the study and numbers who completed the course of evolocumab/placebo are shown separately. ${ }^{\mathrm{a}}$ One of these participants completed placebo but was not reachable for the end of study visit; ${ }^{b}$ discontinued placebo with the reason of 'participant request'

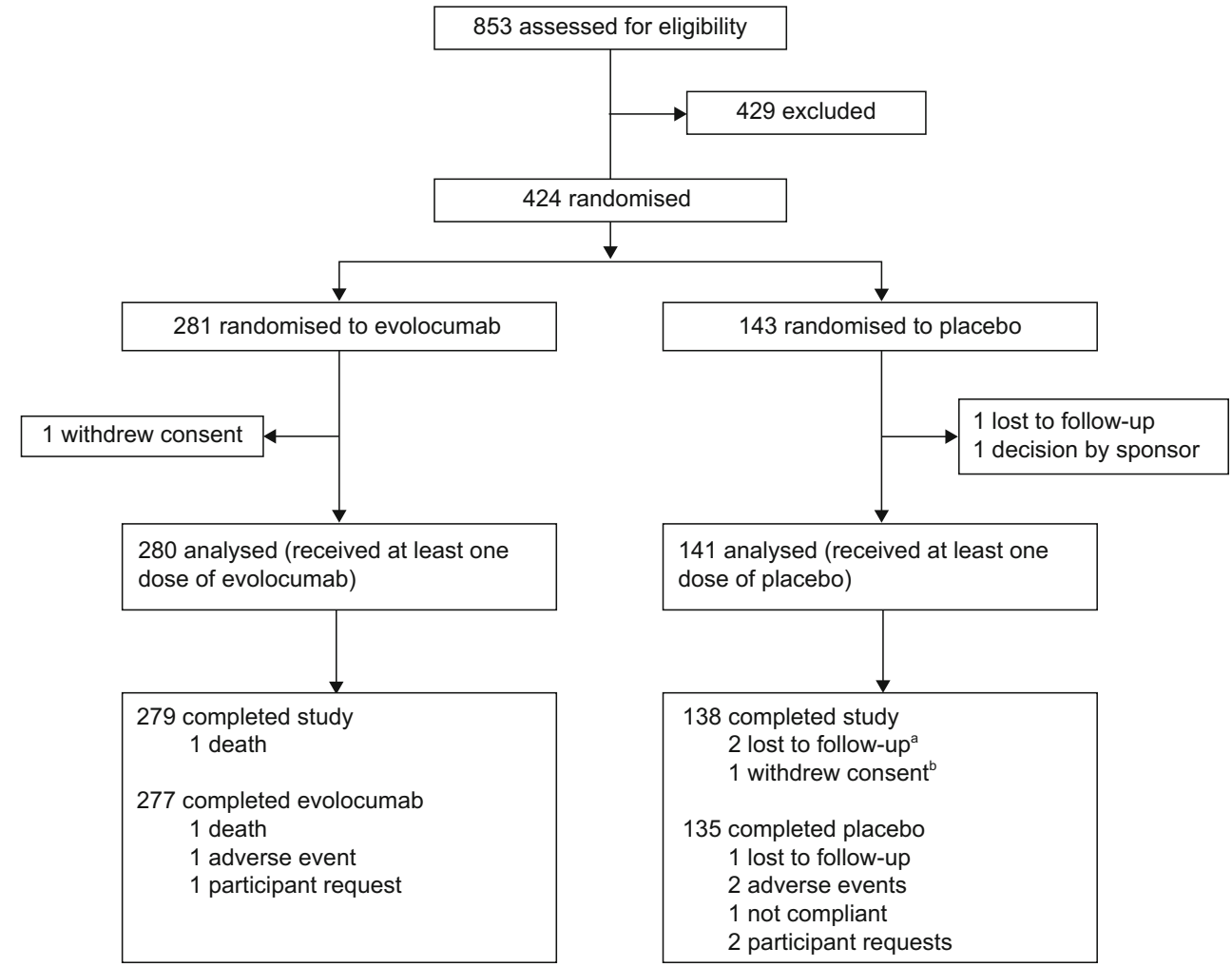


Table 1 Baseline demographics and characteristics of the study population

\begin{tabular}{|c|c|c|}
\hline Characteristic & $\begin{array}{l}\text { Placebo } \\
(n=141)\end{array}$ & $\begin{array}{l}\text { Evolocumab } \\
(n=280)\end{array}$ \\
\hline \multicolumn{3}{|l|}{ Demographic } \\
\hline Sex, female, $n(\%)$ & $65(46.1)$ & $120(42.9)$ \\
\hline Age, years, mean (SD) & $62.2(8.4)$ & $62.5(8.5)$ \\
\hline Race, white, $n(\%)$ & $101(71.6)$ & $222(79.3)$ \\
\hline Ethnicity, not Hispanic/Latino, $n(\%)$ & $117(83.0)$ & $226(80.7)$ \\
\hline \multicolumn{3}{|l|}{ Clinical } \\
\hline Systolic BP, mmHg, mean (SD) & $131.4(17.8)$ & $129.9(14.6)$ \\
\hline BMI, $\mathrm{kg} / \mathrm{m}^{2}$, mean (SD) & $33.1(7.2)$ & $33.4(6.1)$ \\
\hline Waist circumference, $\mathrm{cm}$, mean (SD) & $108.9(16.5)$ & $109.0(15.8)$ \\
\hline \multicolumn{3}{|c|}{ Background lipid therapy per ACC/AHA definition, $n(\%)^{\mathrm{a}}$} \\
\hline High-intensity statin & $72(51.1)$ & $146(52.1)$ \\
\hline Moderate-intensity statin & $67(47.5)$ & $133(47.5)$ \\
\hline Hypertension, $n(\%)$ & $119(84.4)$ & $247(88.2)$ \\
\hline Cerebrovascular or peripheral arterial disease, $n(\%)$ & $33(23.4)$ & $67(23.9)$ \\
\hline Coronary artery disease, $n(\%)$ & $44(31.2)$ & $119(42.5)$ \\
\hline Coronary artery stenosis $>50 \%$ & $23(16.3)$ & $51(18.2)$ \\
\hline Myocardial ischaemia & $15(10.6)$ & $29(10.4)$ \\
\hline Angina pectoris & $16(11.3)$ & $56(20.0)$ \\
\hline Myocardial infarction & $22(15.6)$ & $46(16.4)$ \\
\hline Coronary artery bypass & $13(9.2)$ & $45(16.1)$ \\
\hline Percutaneous coronary intervention & $26(18.4)$ & $63(22.5)$ \\
\hline COPD, $n(\%)$ & $10(7.1)$ & $24(8.6)$ \\
\hline Diabetes-related medication use, $n(\%)$ & $141(100)$ & $280(100)$ \\
\hline Insulin use, $n(\%)$ & $54(38.3)$ & $97(34.6)$ \\
\hline \multicolumn{3}{|l|}{ Lipid values, mean (SD) } \\
\hline LDL-C, mmol/1, mean (SD) & $2.86(0.85)$ & $2.81(0.80)$ \\
\hline Non-HDL-C, mmol/l, mean (SD) & $3.77(0.88)$ & $3.75(0.90)$ \\
\hline ApoB, g/l, mean (SD) & $0.98(0.22)$ & $0.97(0.23)$ \\
\hline Total cholesterol, mmol/l, mean (SD) & $4.94(0.91)$ & $4.88(0.95)$ \\
\hline $\mathrm{Lp}(\mathrm{a}), \mathrm{nmol} / \mathrm{l}$, mean $(\mathrm{SD})$ & $99.40(122.80)$ & $88.00(111.50)$ \\
\hline Triacylglycerol, mmol/l, mean (SD) & $2.00(1.01)$ & $2.08(1.16)$ \\
\hline HDL-C, mmol/l, mean (SD) & $1.17(0.32)$ & $1.13(0.33)$ \\
\hline $\mathrm{HbA}_{1 \mathrm{c}}, \%$, median $(\mathrm{Q} 1, \mathrm{Q} 3)$ & $7.2(6.5,8.2)$ & $7.3(6.5,8.4)$ \\
\hline $\mathrm{HbA}_{1 \mathrm{c}}, \mathrm{mmol} / \mathrm{mol}$, median (Q1, Q3) & $55(48,66)$ & $56(48,68)$ \\
\hline Fasting serum glucose, mmol/l, median $(\mathrm{Q} 1, \mathrm{Q} 3)$ & $7.4(6.0,9.2)$ & $7.7(6.1,9.6)$ \\
\hline
\end{tabular}

${ }^{a}$ Criteria modified from ACC/AHA guidelines: high intensity, atorvastatin 40-80 mg, rosuvastatin 20-40 mg, simvastatin $80 \mathrm{mg}$; moderate intensity, atorvastatin 10-20 mg, rosuvastatin 5-10 mg, simvastatin 20-40 mg, pravastatin 40-80 mg, lovastatin $40 \mathrm{mg}$, fluvastatin XL $80 \mathrm{mg}$, pitavastatin 2-4 mg
LDL-C was $2.81(0.80) \mathrm{mmol} / \mathrm{l}$ in the evolocumab treatment group and $2.86(0.85) \mathrm{mmol} / \mathrm{l}$ in the placebo group. NonHDL-C was $3.75(0.90) \mathrm{mmol} / \mathrm{l}$ in the evolocumab group and $3.77(0.88) \mathrm{mmol} / \mathrm{l}$ in the placebo group. Other lipids were also well matched at baseline between evolocumab and placebo treatment groups.

Changes in lipids and glycaemic measures Versus placebo, evolocumab treatment decreased LDL-C by a mean (SEM) of $53.1 \%(2.3 \%)$ at week 12 and by $64.1 \%(2.1 \%)$ at the mean of weeks 10 and 12 (combined $p<0.0001$ ) (Tables 2 and 3). Compared with the placebo group, more participants in the evolocumab group achieved an LDL-C level $<1.81 \mathrm{mmol} / 1$ (84.5\% vs $15.4 \%$ at week 12 , and $92.7 \%$ vs $14.8 \%$ at the mean of weeks 10 and 12 ; combined $p<0.0001)$. A $\geq 50 \%$ reduction in LDL-C was more common in the evolocumab group vs placebo group $(65.5 \%$ vs $0.8 \%$ at week 12 , and $84.2 \%$ vs $0.7 \%$ at the mean of weeks 10 and 12 ; combined $p<0.0001$ ). 
Table 2 Efficacy results at week 12 and at the mean of weeks 10 and 12

\begin{tabular}{|c|c|c|c|c|}
\hline \multirow[t]{2}{*}{ Variable } & \multicolumn{2}{|l|}{ Week 12} & \multicolumn{2}{|c|}{ Mean of weeks 10 and 12} \\
\hline & $\begin{array}{l}\text { Placebo } \\
(n=141)\end{array}$ & $\begin{array}{l}\text { Evolocumab } \\
(n=280)\end{array}$ & $\begin{array}{l}\text { Placebo } \\
(n=141)\end{array}$ & $\begin{array}{l}\text { Evolocumab } \\
(n=280)\end{array}$ \\
\hline \multicolumn{5}{|l|}{ LDL-C } \\
\hline Change from baseline, $\%$, mean $(\mathrm{SEM})^{\mathrm{a}}$ & $-1.1(1.9)$ & $-54.3(1.4)$ & $-0.8(1.8)$ & $-65.0(1.3)$ \\
\hline Treatment difference, mean $(\mathrm{SEM})^{\mathrm{b}}$ & $-53.1(2.3)^{\dagger}$ & & $-64.1(2.1)^{\dagger}$ & \\
\hline Achievement of $<1.81 \mathrm{mmol} / 1, n(\%)$ & $20(15.4)$ & $213(84.5)$ & $20(14.8)$ & $253(92.7)$ \\
\hline Achievement of $\geq 50 \%$ reduction, $n(\%)$ & $1(0.8)$ & $165(65.5)$ & $1(0.7)$ & $221(84.2)$ \\
\hline \multicolumn{5}{|c|}{ Change from baseline in other lipids, $\%$, mean $(\mathrm{SEM})^{\mathrm{a}}$} \\
\hline Non-HDL-C & $-0.6(1.8)$ & $-46.9(1.3)$ & $-0.1(1.6)$ & $-56.6(1.2)$ \\
\hline ApoB & $1.8(1.7)$ & $-40.3(1.3)$ & $2.3(1.6)$ & $-50.2(1.2)$ \\
\hline Total cholesterol & $-1.2(1.4)$ & $-35.0(1.0)$ & $-1.1(1.2)$ & $-42.2(0.9)$ \\
\hline $\mathrm{Lp}(\mathrm{a})$ & $7.4(3.1)$ & $-25.2(2.3)$ & $9.6(3.3)$ & $-30.9(2.4)$ \\
\hline Triacylglycerol & $4.8(3.4)$ & $-8.9(2.5)$ & $6.6(2.9)$ & $-12.6(2.2)$ \\
\hline HDL-C & $-1.4(1.4)$ & $6.0(1.0)$ & $-2.6(1.3)$ & $7.2(0.9)$ \\
\hline VLDL-C & $3.0(2.9)$ & $-10.3(2.2)$ & $3.4(2.6)$ & $-13.6(1.9)$ \\
\hline \multicolumn{5}{|c|}{ Change from baseline in glycaemic measure, median (Q1, Q3) } \\
\hline $\mathrm{HbA}_{1 \mathrm{c}}, \%$ & $0.1(-0.2,0.5)$ & $0.1(-0.2,0.5)^{ \pm}$ & $\mathrm{N} / \mathrm{A}$ & N/A \\
\hline $\mathrm{HbA}_{1 \mathrm{c}}, \mathrm{mmol} / \mathrm{mol}$ & $1.1(-2.2,5.5)$ & $1.1(-2.2,5.5)$ & & \\
\hline Fasting serum glucose, $\mathrm{mmol} / \mathrm{l}$ & $0.2(-0.8,1.6)$ & $0.3(-0.8,1.7)^{\S}$ & N/A & N/A \\
\hline
\end{tabular}

${ }^{a}$ Least-squares mean is from the repeated-measures model, which includes treatment group, stratification factor (from interactive voice response system), scheduled visit and the interaction of treatment with scheduled visit as covariates

${ }^{\mathrm{b}}$ Treatment differences use s.c. placebo as the reference

${ }^{\dagger} p<0.0001 ;{ }^{\star} p=0.939 ;$ and ${ }^{\S} p=0.785$ all vs placebo

$\mathrm{N} / \mathrm{A}$, not applicable (not measured)

The mean percentage change from baseline in other lipids is reported in Tables 2 and 3. Statistically significant improvements in favour of evolocumab were observed for non-HDLC, ApoB, total cholesterol, Lp(a), triacylglycerol, HDL-C and VLDL-C (all $p<0.0001)$.

At week 12, fasting serum glucose measures changed from a median (quartile 1, quartile 3 [Q1, Q3]) baseline value of 7.7 $(6.1,9.6) \mathrm{mmol} / \mathrm{l}$ to a week 12 value of $8.2(6.5,10.0) \mathrm{mmol} / \mathrm{l}$ for evolocumab, and a median (Q1, Q3) baseline value of 7.4 $(6.0,9.2) \mathrm{mmol} / \mathrm{l}$ to a week 12 value of $7.8(6.3,10.3) \mathrm{mmol} / \mathrm{l}$ for placebo. These corresponded to median (Q1, Q3) changes from baseline to week 12 in fasting serum glucose of 0.3 $(-0.8,1.7) \mathrm{mmol} / \mathrm{l}$ for evolocumab-treated individuals, and $0.2(-0.8,1.6) \mathrm{mmol} / 1$ for placebo-treated individuals (Table 2).

At week 12, $\mathrm{HbA}_{1 \mathrm{c}}$ levels changed from a median $(\mathrm{Q} 1, \mathrm{Q} 3)$ baseline value of $7.3 \%(6.5 \%, 8.4 \%)(56[48,68] \mathrm{mmol} / \mathrm{mol})$ to $7.4 \%(6.7 \%, 8.6 \%)(57[50,70] \mathrm{mmol} / \mathrm{mol})$ for evolocumab and from a median $(\mathrm{Q} 1, \mathrm{Q} 3)$ baseline value of $7.2 \%(6.5 \%$, $8.2 \%)(55[48,66] \mathrm{mmol} / \mathrm{mol})$ to $7.4 \%(6.6 \%, 8.6 \%)(57[49$, $70] \mathrm{mmol} / \mathrm{mol}$ ) for placebo. These corresponded to median (Q1, Q3) changes from baseline to week 12 in $\mathrm{HbA}_{1 \mathrm{c}}$ of $0.1 \%(-0.2 \%, 0.5 \%)(1.1[-2.2,5.5] \mathrm{mmol} / \mathrm{mol})$ for evolocumab-treated participants and $0.1 \%(-0.2 \%, 0.5 \%)$ $(1.1[-2.2,5.5] \mathrm{mmol} / \mathrm{mol})$ for placebo-treated individuals.
Changes in fasting and post-MMTT variables For the exploratory endpoints of percentage change in fasting and postprandial lipid variables from day 1 to week 12 in response to MMTT, significant changes were observed in the evolocumab group for the mean or median AUC (0-120 min; Table 4). Results observed for the mean or median AUC for 0 $180 \mathrm{~min}$ were consistent with those observed for 0-120 min, although not all variables were statistically significant; this may be due, in part, to almost complete clearance of chylomicrons by $120 \mathrm{~min}$ in some participants and the smaller sample sizes at $180 \mathrm{~min}$ (Table 4).

At week 12 after a mixed meal, the median (Q1, Q3) percentage change in AUC (0-120 min) for LDL-C was $-60.6 \%$ $(-71.6 \%,-39.9 \%)$ for evolocumab vs $-2.1 \%(-10.5 \%$, $10.5 \%)$ for placebo; and the mean (SEM) percentage change in AUC (0-120 min) for non-HDL-C was $-42.7 \%$ (1.3\%) for evolocumab vs $2.7 \%$ (2.0\%) for placebo. The median (Q1, Q3) percentage change in AUC (0-120 min) was: for chylomicron cholesterol, $-19.7 \%(-34.1 \%, 0.0 \%)$ for evolocumab vs $-1.8 \%(-16.7 \%, 28.3 \%)$ for placebo; for chylomicron triacylglycerol, $-12.8 \%(-33.8 \%, 25.4 \%)$ for evolocumab vs $6.9 \%(-19.9 \%, 42.5 \%)$ for placebo; and for ApoB-48, $-14.5 \%(-36.9 \%, 18.2 \%)$ for evolocumab vs $0.0 \%(-26.6 \%$, $31.6 \%)$ for placebo. The median (Q1, Q3) percentage change in AUC (0-120 min) for triacylglycerol was $-13.4 \%$ 
Table 3 Treatment difference between evolocumab vs placebo for lipid variables

\begin{tabular}{lll}
\hline Variable & Week 12 & Mean of weeks 10 and 12 \\
\hline Lipid efficacy, mean $(95 \%$ CI) treatment difference & & \\
Change from baseline in LDL-C, $\%$ & $-53.1(-57.6,-48.7)$ & $-64.1(-68.2,-60.1)$ \\
Achievement of LDL-C <1.81 mmol/1, $\%$ & $69.1(60.4,75.7)$ & $77.9(70.0,83.5)$ \\
Achievement of $\geq 50 \%$ reduction in LDL-C, $\%$ & $64.7(57.7,70.3)$ & $83.5(77.7,87.4)$ \\
Per cent change from baseline in other lipids, mean $(95 \%$ CI) treatment difference \\
Non-HDL-C & $-46.3(-50.4,-42.2)$ & $-56.6(-60.3,-52.9)$ \\
ApoB & $-42.1(-46.1,-38.1)$ & $-52.5(-56.1,-48.9)$ \\
Total cholesterol & $-33.7(-36.9,-30.6)$ & $-41.1(-43.9,-38.2)$ \\
Lp(a) & $-32.6(-39.6,-25.5)$ & $-40.5(-48.1,-32.9)$ \\
Triacylglycerol & $-13.7(-21.6,-5.8)$ & $-19.3(-26.0,-12.5)$ \\
HDL-C & $7.4(4.2,10.6)$ & $9.8(7.0,12.6)$ \\
VLDL-C & $-13.3(-20.1,-6.6)$ & $-17.1(-22.9,-11.2)$ \\
\hline
\end{tabular}

Placebo, $n=141$; evolocumab, $n=280$

All adjusted $p$ values for measures reported in the table were $p<0.0001$ for evolocumab vs placebo comparison
$(-26.6 \%, 8.4 \%)$ for evolocumab vs $4.6 \%(-13.0 \%, 29.3 \%)$ for placebo; mean (SEM) percentage change in AUC (0120 min for VLDL-C was $-5.8 \%$ (2.4\%) for evolocumab vs $7.3 \%(3.0 \%)$ for placebo (Table 4$)$. The aforementioned AUC $(0-120)$ results were all significant with $p<0.05$ for evolocumab.

For glucose metabolism measures in response to MMTT, median (Q1, Q3) AUC (0-120 min) were all non-significant: insulin $(0.0 \%$ [-22.0\%, 29.1\%] for evolocumab vs $6.6 \%$ $[-22.5 \%, 50.0 \%]$ for placebo); C-peptide $(2.0 \%[-12.4 \%$, $17.0 \%]$ for evolocumab vs $4.1 \%[-13.8 \%, 22.9 \%]$ for placebo); and glucagon $(-0.1 \%[-20.4 \%, 23.4 \%]$ for evolocumab vs $2.8 \%$ [-22.4\%, 39.2\%] for placebo). Results for additional variables and AUC (0-180 min) can be found in Table 4.

Data are presented in ESM Table 1 for MMTT variables stratified by the baseline median triacylglycerol level of $1.8 \mathrm{mmol} / \mathrm{l}$. Absolute values at week 12 for $0 \mathrm{~min}$ and 120 min timepoints are provided in ESM Table 2.

Safety Treatment-emergent adverse events (AEs) are listed in Table 5. AEs were reported in 110 (39.3\%) evolocumab-treated participants and $52(36.9 \%)$ placebo-treated participants. Serious AEs occurred in 14 (5.0\%) evolocumab-treated participants and two (1.4\%) placebo-treated participants. No serious $\mathrm{AE}$ was considered to be related to the investigational product or to the device. No pattern in the serious AE was identified in either group. Chronic obstructive pulmonary disease (COPD) was the only serious $\mathrm{AE}$ reported by $\geq 1 \%$ of participants in any treatment group ( $1.4 \%$ evolocumab, $0 \%$ placebo). AEs led to study discontinuation in $0.4 \%$ (evolocumab) and $1.4 \%$ (placebo) of participants; none of these AEs was considered serious. The most common AEs occurring in $\geq 2 \%$ of participants in either treatment group are shown in Table 5. AEs reported in $\geq 2 \%$ of participants in the evolocumab group and not in the placebo group were hypertension (3.9\%) and COPD (2.1\%). Ten of the 11 participants reporting an $\mathrm{AE}$ of hypertension had hypertension at baseline and were receiving pharmacological treatment (seven participants were treated with two or more drugs). Despite anti-hypertensive treatment, BP at baseline was $\geq 140$ and/or $90 \mathrm{mmHg}$ in eight participants reporting an $\mathrm{AE}$ of hypertension. Four of the six participants reporting an AE of COPD had COPD at baseline; two participants with a history of COPD were not receiving treatment at baseline; all six were current or former smokers.

\section{Discussion}

Many individuals with type 2 diabetes receiving statin therapy have LDL-C and non-HDL-C levels that exceed recommended lipid levels [10]. Persistent elevations in LDL-C and nonHDL-C may derive from insufficient LDL-C-lowering efficacy with moderate- to high-intensity statins, lack of statin titration in individuals with diabetes mellitus taking a low- to moderate-intensity statin who are not titrated to a highintensity dosage [7] or statin-associated AEs [11]. In a highrisk population of individuals with diabetes who were treated with a high-intensity statin, higher VLDL-C and small cholesterol-enriched VLDL particles were associated with an increased risk of recurrent atherosclerotic cardiovascular disease (ASCVD) events [1].

We investigated the efficacy and safety of evolocumab in individuals with type 2 diabetes who had elevated LDL-C or non-HDL-C levels on a maximally tolerated dose of a statin of at least moderate intensity. Several guidelines advocate more aggressive LDL-C lowering to levels $<1.81 \mathrm{mmol} / 1$ in 
Table 4 Week 12 AUC for MMTT variables

\begin{tabular}{llllll}
\hline \multirow{2}{*}{$\begin{array}{l}\text { Change from baseline } \\
\text { to week 12 AUC }(\%)\end{array}$} & AUC 0-120 min & & & AUC 0-180 min \\
\cline { 2 - 3 } \cline { 5 - 6 } & Placebo & Evolocumab & & Placebo & Evolocumab \\
\hline Total cholesterol & $1.7(1.5)[128]$ & $-30.9(1.0)^{*}[257]$ & & $1.1(2.9)[27]$ & $-31.3(2.3)[57]$ \\
LDL-C & $-2.1(-10.5,10.5)[117]$ & $-60.6(-71.6,-39.9)^{*}[235]$ & & $-2.7(-5.8,3.8)[25]$ & $-59.7(72.8,-37.6)^{*}[53]$ \\
HDL-C & $-0.5(1.1)[128]$ & $8.7(1.0)^{*}[257]$ & & $4.0(2.4)[27]$ & $10.6(2.3)^{*}[57]$ \\
Non-HDL-C & $2.7(2.0)[128]$ & $-42.7(1.3)[257]$ & & $0.5(3.7)[27]$ & $-43.2(2.8)[57]$ \\
Triacylglycerol & $4.6(-13.0,29.3)[128]$ & $-13.4(-26.6,8.4)^{*}[257]$ & & $-4.7(-18.7,36.3)[27]$ & $-17.0(-32.7,7.1)^{*}[57]$ \\
VLDL-C & $7.3(3.0)[117]$ & $-5.8(2.4)^{*}[239]$ & & $6.8(6.8)[25]$ & $-8.3(4.4)[55]$ \\
Chylomicron triacylglycerol & $6.9(-19.9,42.5)[96]$ & $-12.8(-33.8,25.4)^{*}[183]$ & & $-7.3(-15.8,23.0)[25]$ & $-7.4(-25.5,26.1)^{*}[44]$ \\
Chylomicron cholesterol & $-1.8(-16.7,28.3)[97]$ & $-19.7(-34.1,0.0)^{*}[185]$ & & $-4.2(-15.8,28.3)[25]$ & $-24.2(-33.6,-5.4)[44]$ \\
ApoB-48 & $0.0(-26.6,31.6)[125]$ & $-14.5(-36.9,18.2)^{*}[252]$ & & $-0.7(-26.2,34.9)[26]$ & $-8.5(-36.3,23.3)[59]$ \\
Plasma glucose & $2.5(-9.6,22.8)[129]$ & $2.6(-9.0,18.2)[257]$ & & $7.5(-8.0,18.8)[27]$ & $6.6(-8.5,15.6)[60]$ \\
Insulin & $6.6(-22.5,50.0)[126]$ & $0.0(-22.0,29.1)[248]$ & & $14.3(-14.1,53.4)[27]$ & $8.7(-23.1,40.6)[56]$ \\
Proinsulin & $6.7(-18.1,41.6)[128]$ & $0.0(-22.4,39.6)[260]$ & & $5.9(-11.7,49.1)[28]$ & $-2.7(-26.5,19.9)[62]$ \\
C-peptide & $4.1(-13.8,22.9)[125]$ & $2.0(-12.4,17.0)[243]$ & & $-0.1(-5.6,30.1)[25]$ & $2.4(-18.4,11.7)[55]$ \\
Glucagon & $2.8(-22.4,39.2)[120]$ & $-0.1(-20.4,23.4)[242]$ & & $0.5(-18.9,48.2)[25]$ & $-3.3(-23.3,26.1)[57]$ \\
NEFA & $-1.3(-18.8,21.3)[131]$ & $-2.1(-24.0,26.7)[262]$ & & $2.2(-26.8,28.6)[28]$ & $-4.6(-19.6,15.3)[61]$ \\
IL-6 & $-1.6(-21.7,17.4)[127]$ & $-0.8(-21.5,29.5)[259]$ & & $3.8(-11.4,31.0)[28]$ & $-5.8(-25.7,30.9)[59]$ \\
Adiponectin & $-0.8(-12.9,12.3)[130]$ & $-2.1(-16.0,15.0)[262]$ & & $1.7(-11.6,10.2)[28]$ & $1.8(-8.5,17.7)[61]$ \\
\hline
\end{tabular}

All data presented are median (Q1, Q3) AUC [n], except for total cholesterol, HDL-C, non-HDL-C and VLDL-C, which are mean (SEM) AUC [ $n]$ $n$, number of participants in the full analysis set who had MMTT timepoint (120 min) or extended-timepoint (180 min) assessments $* p<0.05$ (nominal, post hoc, no multiplicity adjustment)

individuals with ASCVD or $<2.6 \mathrm{mmol} / \mathrm{l}$ in individuals with diabetes. In our study, addition of evolocumab to background statin therapy greatly reduced LDL-C levels vs placebo and enabled most individuals to reach LDL-C levels $<1.81 \mathrm{mmol} / \mathrm{l}$. The ACC/AHA has adopted a desired LDL-C-lowering efficacy of $>50 \%$ [12], with an updated decision pathway recommending a $>50 \%$ reduction in LDL-C and consideration of non-statin therapies such as ezetimibe or a proprotein convertase subtilisin/kexin type 9 inhibitor (PCSK9i) when additional LDL-C lowering is desired. Guidelines from the American Association of Clinical Endocrinologists (AACE) and ADA also recommend additional non-statin therapy for high-risk individuals who do not achieve an effective $>50 \%$ reduction in LDL-C [2] or desired LDL-C levels [13, 14] with maximally tolerated statin. In the BANTING trial, we observed that a substantially greater proportion of participants randomised to evolocumab therapy vs placebo achieved $\geq 50 \%$ reduction in LDL-C. The shorter duration of follow-up (12 weeks) limited the ability to assess long-term safety and durability of response.

FOURIER included 11,031 (40\%) participants with diabetes who received treatment with moderate- to high-intensity statin over a median follow-up of 26 months $[8,15]$. The HR for the primary cardiovascular endpoint (cardiovascular death, MI, stroke, hospitalisation for unstable angina, or coronary revascularisation) in participants with diabetes was 0.83
(95\% CI $0.75,0.93 ; p=0.0008)$ and $0.87(0.79,0.96 ; p=$ $0.0052)$ for participants without diabetes $\left(p_{\text {interaction }}=0.64\right)$ [8]. The HR for the secondary endpoint was $0.82(0.72$, $0.93 ; p=0.0021)$ for individuals with diabetes and 0.78 $(0.69,0.89 ; p=0.002)$ for those without diabetes [8]. Because of the higher baseline cardiovascular risk of individuals with type 2 diabetes, the absolute risk reduction in the primary endpoint with evolocumab tended to be greater in those with diabetes $(2.7 \%$ [95\% CI $0.7,4.8])$ vs those without diabetes $(1.6 \%[0.1,3.2])$ over 3 years [8].

Non-HDL-C is more strongly associated with ASCVD events than LDL-C in participants with diabetes, and it is a co-primary or secondary target of cholesterol-lowering therapies $[13,14]$. Here, in the BANTING study, we evaluated the efficacy of evolocumab therapy on non-HDL-C levels and on the achievement of non-HDL-C levels that are recommended by several guidelines. Evolocumab reduced non-HDL-C levels by $47 \%$ vs $1 \%$ reduction with placebo at week 12 , and by $57 \%$ vs $1 \%$ reduction with placebo at the mean of weeks 10 and 12. Evolocumab also improved levels of other lipid and lipoprotein fractions.

No notable effects in response to MMTT were observed in the evolocumab treatment group for glucose metabolism measures (plasma glucose, insulin, proinsulin, C-peptide, glucagon and NEFA) (median AUC 0-120 min and/or median AUC 0-180 min) (Table 4). The reductions we observed in 
Table 5 Safety

\begin{tabular}{|c|c|c|}
\hline $\mathrm{AE}$ & $\begin{array}{l}\text { Placebo }(n=141) \\
n(\%)\end{array}$ & $\begin{array}{l}\text { Evolocumab }(n=280) \\
n(\%)\end{array}$ \\
\hline Treatment-emergent & $52(36.9)$ & $110(39.3)$ \\
\hline Serious $^{\mathrm{a}}$ & $2(1.4)$ & $14(5.0)$ \\
\hline Leading to discontinuation of evolocumab or placebo & $2(1.4)$ & $1(0.4)$ \\
\hline Serious & $0(0.0)$ & $0(0.0)$ \\
\hline Non-serious & $2(1.4)$ & $1(0.4)$ \\
\hline Fatal AEs ${ }^{\mathrm{b}}$ & $0(0.0)$ & $1(0.4)$ \\
\hline \multicolumn{3}{|l|}{ Most common ${ }^{\mathrm{c}}$} \\
\hline Hypertension & $0(0.0)$ & $11(3.9)$ \\
\hline Diabetes mellitus $^{\mathrm{d}}$ & $5(3.5)$ & $8(2.9)$ \\
\hline Diarrhoea & $4(2.8)$ & $6(2.1)$ \\
\hline Headache & $3(2.1)$ & $6(2.1)$ \\
\hline Urinary tract infection & $2(1.4)$ & $6(2.1)$ \\
\hline COPD & $0(0.0)$ & $6(2.1)$ \\
\hline Viral upper respiratory tract infection & $4(2.8)$ & $5(1.8)$ \\
\hline Pharyngitis & $3(2.1)$ & $0(0.0)$ \\
\hline Back pain & $3(2.1)$ & $2(0.7)$ \\
\hline \multicolumn{3}{|l|}{ Abnormal laboratory tests } \\
\hline $\mathrm{CK}>5 \times \mathrm{ULN}$ & $0(0.0)$ & $1(0.4)^{\mathrm{e}}$ \\
\hline $\mathrm{CK}>10 \times \mathrm{ULN}$ & $0(0.0)$ & $1(0.4)^{\mathrm{e}}$ \\
\hline $\mathrm{AST}$ or $\mathrm{ALT}>3 \times \mathrm{ULN}$ & $1(0.7)$ & $1(0.4)$ \\
\hline
\end{tabular}

${ }^{a} \mathrm{COPD}$ was the only $\mathrm{AE}$ reported by $\geq 1 \%$ of participants in any treatment group (1.4\% evolocumab, $0 \%$ placebo). Four COPD, two coronary artery disease and one hypertension serious AE were preceded by the same disease history (i.e. the COPD event was preceded by history of COPD) at baseline. One participant in the evolocumab group experienced four serious AEs (COPD, bacterial pneumonia, sepsis and dehydration). One participant in the evolocumab group with a history of coronary artery disease, MI, peripheral arterial disease (PAD) and stroke experienced a fatal $\mathrm{AE}$ of sudden cardiac death; this event was not considered related to the investigational product by the investigator

${ }^{\mathrm{b}}$ Sudden cardiac death 8 days after exposure to evolocumab; not considered related to evolocumab by investigator

${ }^{\mathrm{c}}$ Reported in $\geq 2 \%$ of participants in one or more treatment groups

${ }^{\mathrm{d}}$ Worsening of diabetes or diabetes control per investigator

${ }^{\text {e }}$ Participant reported vigorous exercise prior to the week 12/end of study visit

ALT, alanine aminotransferase; AST, aspartate aminotransferase; CK, creatine kinase; ULN, upper limit of normal

postprandial triacylglycerol-rich lipoproteins such as VLDLC, chylomicron triacylglycerol and ApoB-48 were consistent with the concept that remnant lipoproteins are cleared, in part, by the LDL receptor. Our results differ from a recent analysis from the Effects on Lipoprotein Metabolism From PCSK9 Inhibition Utilizing a Monoclonal Antibody (FLOREY) study, where postprandial levels of chylomicrons and triacylglycerol were not altered in healthy men with LDL-C $>2.59 \mathrm{mmol} / \mathrm{l}$ treated with evolocumab [16]. However, the FLOREY analysis was conducted in a small number of normolipidaemic men. Our results here are largely consistent with those of other phase II and III studies of evolocumab, demonstrating that evolocumab treatment leads to modest reductions in triacylglycerol $[17,18]$.

In this study of participants with type 2 diabetes, there were no notable differences in fasting glucose or $\mathrm{HbA}_{1 \mathrm{c}}$ levels between treatment arms at the end of study. At $2 \mathrm{~h}$ after a mixed meal, there were no differences between treatment arms in glucose, insulin, proinsulin, C-peptide, glucagon or NEFA concentrations. These data support the short-term safety of evolocumab on glycaemic variables in individuals with type 2 diabetes.

In FOURIER, levels of $\mathrm{HbA}_{1 \mathrm{c}}$ and fasting plasma glucose were similar over time in both the evolocumab and placebo groups in participants with or without diabetes, and evolocumab did not increase the risk of new-onset diabetes in participants without diabetes (HR 1.05; 95\% CI 0.94, 1.17), including those participants with impaired fasting glucose at baseline (HR 1.00; 95\% CI 0.89, 1.13). In participants with diabetes at baseline, incidences of AEs and serious AEs were similar between placebo and evolocumab treatment groups in FOURIER, and imbalances between these treatment groups were not observed for the incidence of hypertension or COPD AEs [8]. The safety and efficacy of the PCSK9i alirocumab in individuals with diabetes has been evaluated in several multicentre trials. Safety and 
changes in lipid efficacy were similar in individuals with insulintreated diabetes, regardless of diabetes type [19], and alirocumab had no effect on the transition to new-onset diabetes in individuals without diabetes at baseline [20,21]. These reports have not indicated any imbalances between treatment groups in the incidence of hypertension or COPD.

In conclusion, in statin-treated individuals with type 2 diabetes and hypercholesterolaemia or mixed dyslipidaemia, the addition of evolocumab compared with placebo resulted in LDL-C reductions of up to $64 \%$ and LDL-C levels $<1.81 \mathrm{mmol} / 1$ in up to $93 \%$ of individuals. In this patient population, the addition of evolocumab compared with placebo resulted in non-HDL-C reductions of up to $57 \%$. Evolocumab treatment had no notable effect on glucose measures and was safe and well tolerated overall.

Acknowledgements The authors acknowledge A. M. Nawrocki of Amgen Inc. for providing editorial support and Ellen Bradley of Amgen Ltd. for providing statistical support. Some of the data from this manuscript was presented, in part, at the ADA 78th Scientific Sessions in Orlando, June 2018. Site investigators by country are listed in the ESM.

Data availability Qualified researchers may request data from Amgen clinical studies. Complete details are available at www.amgen.com/ datasharing.

Funding Amgen Inc. funded this study.

Duality of interest RSR reports: research grants to his institution from Akcea, Amgen, AstraZeneca, CVS Caremark, The Medicines Company, Regeneron and Sanofi; advisory boards for Amgen, Regeneron and Sanofi; honoraria from Akcea, Kowa and Pfizer; stock holdings in MediMergent; and royalties from UpToDate Inc. Neither HB nor his affiliated research centre own pharmaceutical stocks or patents. In the past 12 months, HB's research site has received research grants from Amarin, Amgen, Alere, Allergan, Arisaph, AstraZeneca, Boehringer Ingelheim, Bristol-Myers Squibb, Catabasis, Dr. Reddy's, Eisai, Elcelyx, Lilly, Esperion, Ferrer/Chiltern, Gemphire, Gilead, GlaxoSmithKline, iSpecimen, Janssen, Johnson \& Johnson, Kowa, Merck, Nektar, Nichi-Iko, Novartis, Novo Nordisk, Pfizer, Regeneron, Sanofi, Selecta, Takeda and TIMI. In the past 12 months, HB has served as a consultant/advisor for Alnylam, Akcea, Amgen, AstraZeneca, Eisai, Lilly, Esperion, Ionis (ISIS), Janssen, Johnson \& Johnson, Kowa, Merck, Novartis, ProSciento, Regeneron and Sanofi. In the past 12 months, HB has served as a speaker for Amarin, Amgen, Eisai, Kowa, Orexigen, Regeneron and Sanofi. MLD is a member of the Repatha LCM Scientific Advisory Board. MLM is currently employed by Amgen Inc. and holds Amgen stock/stock options. ME-D is currently employed by Amgen Ltd. and holds Amgen stock/stock options. PP reports: advisory board/lecture honoraria from Lilly, Sanofi and MSD; participation in clinical trials for Lilly, Janssen, Lexicon, AstraZeneca, MSD, Boehringer Ingelheim and GlaxoSmithKline; and research funding or grants from Lilly, MSD, Sanofi, AstraZeneca, Lexicon and Janssen. RS was previously employed by Amgen, holds Amgen stock/stock options and is an inventor on at least one pending patent application owned by Amgen Inc. related to evolocumab. YH reports research grants and consultant and speaker honoraria from Aegerion, Amarin, Amgen, AstraZeneca, Bristol-Myers Squibb, Boehringer Ingelheim, Boehringer IngelheimLilly, Eisai, Gan \& Lee, Grifols, Hanmi, Intarcia, Janssen, Lexicon, Lilly, Merck, Merck-Pfizer, Novo Nordisk, Regeneron and Sanofi. PR reports: research grants from Novo Nordisk and (in the past 12 months) grants from Bristol-Myers Squibb; and consulting with Sanofi.
Contribution statement RSR drafted the manuscript with input from all authors. ME-D conducted statistical analyses. All authors contributed to the interpretation of data and reviewed and approved the final manuscript. RSR is the guarantor of this work. The study sponsor was involved in the design of the study; the collection, analysis, and interpretation of data; the provision of editorial support; and the decision to submit the report for publication.

Open Access This article is distributed under the terms of the Creative Commons Attribution 4.0 International License (http:// creativecommons.org/licenses/by/4.0/), which permits unrestricted use, distribution, and reproduction in any medium, provided you give appropriate credit to the original author(s) and the source, provide a link to the Creative Commons license, and indicate if changes were made.

\section{References}

1. Lawler PR, Akinkuolie AO, Harada P et al (2017) Residual risk of atherosclerotic cardiovascular events in relation to reductions in very-low-density lipoproteins. J Am Heart Assoc 6:e007402

2. Lloyd-Jones DM, Morris PB, Ballantyne CM et al (2017) 2017 focused update of the 2016 ACC Expert Consensus Decision Pathway on the role of non-statin therapies for LDL-cholesterol lowering in the management of atherosclerotic cardiovascular disease risk: a report of the American College of Cardiology Task Force on Expert Consensus Decision Pathways. J Am Coll Cardiol 70(14):1785-1822. https://doi.org/10.1016/j.jacc.2017.07. 745

3. Cholesterol Treatment Trialists' (CTT) Collaborators, Kearney PM, Blackwell L et al (2008) Efficacy of cholesterol-lowering therapy in 18,686 people with diabetes in 14 randomised trials of statins: a meta-analysis. Lancet 371(9607):117-125. https://doi.org/10.1016/ S0140-6736(08)60104-X

4. Mukamal KJ, Nesto RW, Cohen MC et al (2001) Impact of diabetes on long-term survival after acute myocardial infarction: comparability of risk with prior myocardial infarction. Diabetes Care 24(8): 1422-1427. https://doi.org/10.2337/diacare.24.8.1422

5. Giugliano RP, Cannon CP, Blazing MA et al (2018) Benefit of adding ezetimibe to statin therapy on cardiovascular outcomes and safety in patients with versus without diabetes mellitus: results from IMPROVE-IT (Improved Reduction of Outcomes: Vytorin Efficacy International Trial). Circulation 137(15):1571-1582. https://doi.org/10.1161/CIRCULATIONAHA.117.030950

6. Schmidt AF, Swerdlow DI, Holmes MV et al (2017) PCSK9 genetic variants and risk of type 2 diabetes: a mendelian randomisation study. Lancet Diabetes Endocrinol 5(2):97-105. https://doi.org/10. 1016/S2213-8587(16)30396-5

7. Giustino G, Colantonio LD, Brown TM et al (2018) Titration to high-intensity statin therapy following acute myocardial infarction in patients with and without diabetes mellitus. Cardiovasc Drugs Ther 32(5):453-461. https://doi.org/10.1007/s10557-018-6816-8

8. Sabatine MS, Leiter LA, Wiviott SD et al (2017) Cardiovascular safety and efficacy of the PCSK9 inhibitor evolocumab in patients with and without diabetes and the effect of evolocumab on glycaemia and risk of new-onset diabetes: a prespecified analysis of the FOURIER randomised controlled trial. Lancet Diabetes Endocrinol 5(12):941-950. https://doi.org/10.1016/S22138587(17)30313-3

9. Kasichayanula S, Grover A, Emery MG et al (2018) Clinical pharmacokinetics and pharmacodynamics of evolocumab, a PCSK9 inhibitor. Clin Pharmacokinet 57(7):769-779. https://doi.org/10. 1007/s40262-017-0620-7 
10. Leiter LA, Lundman P, da Silva PM et al (2011) Persistent lipid abnormalities in statin-treated patients with diabetes mellitus in Europe and Canada: results of the Dyslipidaemia International Study. Diabet Med 28(11):1343-1351. https://doi.org/10.1111/j. 1464-5491.2011.03360.x

11. Rosenson RS, Baker S, Banach M et al (2017) Optimizing cholesterol treatment in patients with muscle complaints. J Am Coll Cardiol 70(10):1290-1301. https://doi.org/10.1016/j.jacc.2017.07. 752

12. Stone NJ, Robinson JG, Lichtenstein AH et al (2014) 2013 ACC/ AHA guideline on the treatment of blood cholesterol to reduce atherosclerotic cardiovascular risk in adults: a report of the American College of Cardiology/American Heart Association Task Force on Practice Guidelines. J Am Coll Cardiol 63(25): 2889-2934. https://doi.org/10.1016/j.jacc.2013.11.002

13. Jellinger PS, Handelsman Y, Rosenblit PD et al (2017) American Association of Clinical Endocrinologists and American College of Endocrinology Guidelines for Management of Dyslipidemia and Prevention of Cardiovascular Disease. Endocr Pract 23(Suppl 2):187. https://doi.org/10.4158/EP171764.APPGL

14. American Diabetes Association (2018) 15. Diabetes Advocacy: Standards of Medical Care in Diabetes-2018. Diabetes Care 41(Suppl 1):S152-S153. https://doi.org/10.2337/dc18-S015

15. Sabatine MS, Giugliano RP, Keech AC et al (2017) Evolocumab and clinical outcomes in patients with cardiovascular disease. $\mathrm{N}$ Engl J Med 376(18):1713-1722. https://doi.org/10.1056/ NEJMoa1615664

16. Chan DC, Watts GF, Somaratne R, Wasserman SM, Scott R, Barrett PHR (2018) Comparative effects of PCSK9 (proprotein convertase subtilisin/kexin type 9) and statins on postprandial triglyceride-rich lipoprotein metabolism. Arterioscler Thromb Vasc Biol 38(7): 1644-1655. https://doi.org/10.1161/ATVBAHA.118.310882

17. Koren MJ, Lundqvist P, Bolognese $M$ et al (2014) Anti-PCSK9 monotherapy for hypercholesterolemia: the MENDEL-2 randomized, controlled phase III clinical trial of evolocumab. J Am Coll Cardiol 63(23):2531-2540. https://doi.org/10.1016/j.jacc.2014.03. 018

18. Koren MJ, Scott R, Kim JB et al (2012) Efficacy, safety, and tolerability of a monoclonal antibody to proprotein convertase subtilisin/ kexin type 9 as monotherapy in patients with hypercholesterolaemia (MENDEL): a randomised, double-blind, placebo-controlled, phase 2 study. Lancet 380(9858):1995-2006. https://doi.org/10.1016/ S0140-6736(12)61771-1

19. Leiter LA, Müller-Wieland D, Baccara-Dinet MT, Letierce A, Samuel R, Cariou B (2018) Efficacy and safety of alirocumab in people with prediabetes vs those with normoglycaemia at baseline: a pooled analysis of 10 phase III ODYSSEY clinical trials. Diabet Med 35(1):121-130. https://doi.org/10.1111/dme.13450

20. Colhoun HM, Ginsberg HN, Robinson JG et al (2016) No effect of PCSK9 inhibitor alirocumab on the incidence of diabetes in a pooled analysis from 10 ODYSSEY phase 3 studies. Eur Heart $\mathbf{J}$ 37(39):2981-2989. https://doi.org/10.1093/eurheartj/ehw292

21. Leiter LA, Cariou B, Müller-Wieland D et al (2017) Efficacy and safety of alirocumab in insulin-treated individuals with type 1 or type 2 diabetes and high cardiovascular risk: the ODYSSEY DMINSULIN randomized trial. Diabetes Obes Metab 19(12):17811792. https://doi.org/10.1111/dom.13114

Publisher's note Springer Nature remains neutral with regard to jurisdictional claims in published maps and institutional affiliations. 